\title{
Polar interface optical phonon states and their dispersive properties of a wurtzite GaN quantum dot: quantum size effect
}

\author{
L. Zhand* \\ Department of Mechanism and Electronic, Guangzhou Panyu Polytechnic, \\ Guangzhou, 511483, People's Republic of China
}

Received April 23, 2009, in final form September 2, 2009

\begin{abstract}
Based on the macroscopic dielectric continuum model, the interface-optical-propagating (IO-PR) mixing phonon modes of a quasi-zero-dimensional (Q0D) wurtzite cylindrical quantum dot (QD) structure are derived and studied. The analytical phonon states of IO-PR mixing modes are given. It is found that there are two types of IO-PR mixing phonon modes, i.e. $\rho$-IO/z-PR mixing modes and the $z-1 \mathrm{O} / \rho-\mathrm{PR}$ mixing modes existing in QOD wurtzite QDs. Each IO-PR mixing mode also has symmetrical and antisymmetrical forms. Via a standard procedure of field quantization, the Fröhlich Hamiltonians of electron-(IO-PR) mixing phonons interaction are obtained. The orthogonal relations of polarization eigenvectors for these IO-PR mixing modes are also displayed. Numerical calculations for a wurtzite GaN cylindrical QD are focused on the quantum size effect on the dispersive properties of IO-PR mixing modes. The results reveal that both the radial-direction size and the axial-direction size have great effect on the dispersive frequencies of the IO-PR mixing phonon modes. The limiting features of dispersive curves of these phonon modes are discussed in depth. The phonon modes "reducing" the behavior of wurtzite quantum confined structures have been explicitly observed in the systems. Moreover, the behaviors that the IO-PR mixing phonon modes in wurtzite Q0D QDs reduce to the IO modes and PR modes in wurtzite Q2D QW and Q1D QWR systems are profoundly analyzed both from the viewpoint of physics and mathematics. These results show that the present theories of polar mixing phonon modes in wurtzite cylindrical QDs are consistent with the phonon modes theories in wurtzite QWs and QWR systems. The analytical electron-phonon interaction Hamiltonians obtained here are useful in further analyzing the phonon effect on optoelectronic properties of wurtzite Q0D QD structures.
\end{abstract}

Key words: wurtzite nitride quantum dots, phonon states, electron-phonon interactions, quantum size effect

PACS: 81.05.Ea, 78.67.Hc, 63.22.-m, 63.20.Kd

\section{Introduction}

Thanks to the excellent characteristics of wide band-gap, strong atomic bonding and high electronic mobility as well as high optical efficiency, GaN-based semiconductor materials are quite attractive materials as a basis for the creation of reliable high-power or high-temperature electronic equipment and short wave-length optoelectronic devices [1 14]. Following this trend, the investigations on various physical properties of nitride semiconductor low-dimensional quantum structures, such as quasi-2-dimensional (Q2D) quantum wells (QWs) [2 8], quasi-1-dimensional (Q1D) quantum wires (QWRs) 9 16] have become a hot topic. Among these research attempts, the crystal lattice dynamical properties of GaN-based quantum structures have attracted special attention both from theoretical and experimental viewpoint [2-16]. The driving force behind these efforts lies in the evident fact that lattice vibrations have an important effect on the optoelectronic and electronic properties of nitride low-dimensional quantum systems [6 -13]. In fact, the phenomena of phonon replicas in the emission spectra, the homogeneous broadening of excitonic line width and the relaxations of hot carriers to the fundamental band edge are directly related to the lattice vibration of nitride materials [14]. Hence, lattice dynamical properties of nitride low-dimensional quantum structures, especially the Q2D QW and Q1D QWR structures have been intensively studied for the last two decades [2 16].

\footnotetext{
*E-mail: zhangli-gz@263.net
} 
With the technological advancement of the crystal growing, not only Q2D nitride QW and Q1D nitride QWR structures, but also quasi-0-dimensional (Q0D) nitride quantum dots (QDs) can be fabricated [17 27]. It is well known that group-III nitride usually crystallizes in the hexagonal wurtzite structure, whose physical behavior is anisotropic in space. The previous works on the wurtzite QW and QWR structures reveal that the lattice vibrating modes (i.e. phonon modes) and electron-phonon interactions become more complicated with the increase of confined dimensionality [2 10]. Though the bound electronic states, excitonic states, donor bound excitons, as well as the nonlinear optical properties in the Q0D wurtzite QD have been widely investigated [23 27], the polar optical phonon states and their coupling features with electrons in wurtzite Q0D QD systems have rarely been reported due to the complexity of phonon modes in the structures originated from the high confined dimensionality and anisotropic wurtzite structure [28 30]. Fonoberov et al. 28] derived an integral equation that defines interface optical (IO) and confined modes in wurtzite nanocrystals. The spectrum of polar optical-phonon modes is calculated numerically and discussed only on a wurtzite spheroidal nanocrystal. Chassaing and coworkers [29] analyzed the surface optical phonon modes in a wurtzite cylindrical ZnO QD structure, and two types of surface phonon modes, i.e. side surface phonon modes and top surface phonon modes are found. In their studies, infinite height (radius) for the side (top) surface phonon modes were assumed. With the aid of the DCM and Loudon's uniaxial crystal model [31], we extended the works of polar optical phonon modes from the wurtzite Q2D QWs and Q1D QWRs to the wurtzite Q0D QDs structures [30]. However, the dispersive properties of phonon modes in the wurtzite QDs have not been discussed, and electron-phonon interaction Hamiltonian has not been established so far. Thus, we will investigate the polar phonon states and their dispersive properties of a wurtzite Q0D QD, and we will lay emphasis on the quantum size effect of the dispersive spectra of polar phonon modes in the Q0D structure in the present paper.

In fact, the size-dependence of phonon spectra of CdSe/Te and AlGaAs QDs have been widely reported [32 39]. Paula's group 32] experimentally studied the phonon spectra of CdTe QDs as a function of the QD size by means of resonant Raman scattering measurements. Their results show that, as the QD size decreases, the surface optical (SO) modes scattering intensity increases, but the electron-phonon coupling decreases. Hwang et al. [33] and Baranov et al. [34] investigated the effect of quantum size on the polar optical phonon modes in CdSe QDs. The blue-shift and broadening of the SO phonon frequency were clearly observed as the QD size was reduced. Dzhagan et al. [35] analyzed the size effects on the dispersive features of phonon modes in CdSe nanoparticles by using the resonant Raman scattering technology. Recently, Lange et al. [36] experimentally studied the geometry dependence of the first-order Raman scattering band of CdSe nanorod. They observed an explicit frequency blue-shift of longitudinal optical (LO)-like phonon modes as the diameter of the nanorod increases. Comas and coauthors [37] theoretically deduced and analyzed the SO phonon modes in spherical nanostructured QDs and semiconductor quantum rods under the standard dielectric continuum (DC) approach. Their discussions were mainly focused on the dispersion of SO modes as functions of the QD size and dielectric constants $\epsilon_{d}$ of matrix. Vasilevskiy [38] discussed the dispersion frequency of the dipolar vibrational modes versus the radius in a CdSe QD embedded in different nonpolar matrix. Kanyinda-Malu and Cruz [39] investigated the oscillation spectra of IO and LO phonon modes as a function of the radius in AlGaAs cylindrical QDs.

However, up to now, there has been little research into the size dependence of polar vibration spectra in wurtzite quantum systems [40], especially for the new synthesized wurtzite Q0D QDs [1723]. Furthermore, the calculations of polaronic effects in Q0D QD revealed that the quantum-size can greatly effect the binding energy of the polarons in Q0D QD. Moreover, due to the reduction of the dimensionality and the anisotropy of Q0D wurtzite structures, the properties of optical phonon modes in wurtzite QDs should have more distinct phonon branches [24 29]. Hence, it is of vital importance to investigate the size-dependence phonon spectra in Q0D wurtzite QDs.

The main accomplishments and significance of this work are as follows. (i) Based on the DC model (DCM) and Loudon's uniaxial crystal model, the explicit phonon states and dispersive equations of important optical phonon modes, i.e. the IO-propagating (PR) phonon mixing modes 
in wurtzite cylindrical GaN-based QDs are given. The difference and relationship of the phonon states as well as dispersive equations in wurtzite quantum structures with three different confined dimensionality (i.e. QWs, QWRs and QDs) are profoundly analyzed both from the viewpoint of physics and mathematics. (ii) Numerical calculations on the size-dependence dispersive relation of the IO-PR mixing phonon modes are performed, and their characteristics are discussed in detail. Both the radial- and axial- direction size effects are discussed. The limiting behavior of the IO-PR mixing phonon modes as the radial- and axial- direction sizes approach infinity is profoundly analyzed from the viewpoint of physics and mathematics, and a detailed comparison with Q2D QWs and Q1D QWRs 22 5, 9, 10] is also carried out. (iii) The orthogonal relation of polarization eigenvector of IO-PR mixing phonon modes are obtained, and the Fröhlich electron-phonon interaction Hamiltonians are also deduced using the method of field quantization. The analytical electronphonon coupling functions are important and useful for further investigation of polaronic effect on the electronic and optical properties in wurtzite Q0D QD structures. The paper consists of four sections and these are as follows: in section 2, the phonon states of IO-PR mixing modes and their dispersive equations are presented, and the orthogonal relation of polarization eigenvector as well as the Fröhlich electron-phonon interaction Hamiltonians are deduced; in section 3, the numerical calculations on the dispersive frequency of two types of IO-PR mixing modes are carried out and discussed; and finally, we summarize the main results obtained in the paper in section 4 .

\section{Theory}

It is well known that the wurtzite GaN QDs are frequently of different shapes and symmetries, such as spherical-caps, spheroids, hexagonal pyramids and cylindroid structures [17 23], which strongly depends on the material nature and the conditions of growth. For simplicity, we consider a freestanding wurtzite cylindrical QD structure with radius $R$ and height $2 d$ along the $z$-direction. The $z$-axis is taken to be along the direction of the $c$-axis of the wurtzite material and denotes the radial- (axial-) direction as $t(z)$. Thus, under the cylindrical coordinate, the heterointerfaces of the wurtzite QD in $z$-direction are located at $z= \pm d$, and in radius the heterointerface is at $\rho=R$. The frequency-dependent dielectric functions in $t$-direction and $z$-direction are given by

$$
\epsilon_{t}(\omega)=\epsilon_{t}^{\infty} \frac{\omega^{2}-\omega_{t, L}^{2}}{\omega^{2}-\omega_{t, T}^{2}}, \quad \epsilon_{z}(\omega)=\epsilon_{z}^{\infty} \frac{\omega^{2}-\omega_{z, L}^{2}}{\omega^{2}-\omega_{z, T}^{2}} .
$$

Here $\omega_{z, L}, \omega_{z, T}, \omega_{t, L}$ and $\omega_{t, T}$ are the zone center characteristic frequencies of $\mathrm{A}_{1}(\mathrm{LO}), \mathrm{A}_{1}(\mathrm{TO})$, $\mathrm{E}_{1}(\mathrm{LO})$, and $\mathrm{E}_{1}(\mathrm{TO})$ modes, respectively.

\subsection{Phonon states of IO-PR mixing modes and their dispersive equations of GaN cylindri- cal QDs}

Under the DCM and considering the case of free oscillations (the charge density $\rho_{0}(\mathbf{r})=0$ ), the electric displacement vector $\mathbf{D}$ of phonon modes in wurtzite QD satisfies the relation,

$$
\begin{aligned}
\nabla \cdot \mathbf{D} & =-\nabla^{2}[\epsilon(\omega) \Phi(\mathbf{r})] \\
& =-\varepsilon_{0}\left\{\epsilon_{t}(\omega)\left[\frac{1}{\rho} \frac{\partial}{\partial \rho}\left(\rho \frac{\partial}{\partial \rho}\right)+\frac{1}{\rho^{2}} \frac{\partial^{2}}{\partial \varphi^{2}}\right]+\epsilon_{z}(\omega) \frac{\partial^{2}}{\partial z^{2}}\right\} \Phi(\mathbf{r})=0 .
\end{aligned}
$$

Based on the Loudon's uniaxial crystal modes [31] and the Laplace equation (2) in wurtzite crystal, it can be confirmed that there are four types of polar mixing phonon modes in wurtzite cylindrical QD structures [30]. As the first step of solving the complicated mixing optical phonon modes in wurtzite QDs, we will pay attention to the IO-PR mixing phonon modes hereinafter.

The IO-PR mixing mode is a mode which behaves as IO mode in $t(z)$-direction, and behaves as PR mode in $z(t)$-direction. Considering the exchange of $t$-direction and $z$-direction, it is found that the IO-PR mixing modes are also of two forms, i.e. the $z-\mathrm{IO} / \rho-\mathrm{PR}$ and $\rho-\mathrm{IO} / z-\mathrm{PR}$ mixing 
modes. Under the cylindrical coordinates, the electrostatic potential functions of $z-\mathrm{IO} / \rho-\mathrm{PR}$ mixing modes are given by

$$
\begin{aligned}
& \Phi_{m}^{z-\mathrm{IO} / \rho-\mathrm{PR}}(\mathbf{r})=\mathrm{e}^{\mathrm{i} m \varphi} f^{\mathrm{PR}}(\rho) \phi^{\mathrm{IO}}(z), \\
& f^{\mathrm{PR}}(\rho)=\left\{\begin{array}{ll}
a_{1} J_{m}\left(k_{t 1} \rho\right) & \rho \leqslant R \\
a_{2} J_{m}\left(k_{t 2} \rho\right)+a_{3} Y_{m}\left(k_{t 2} \rho\right) & \rho>R
\end{array},\right. \\
& \phi^{\mathrm{IO}}(z)= \begin{cases}\left\{\begin{array}{ll}
b_{1} \exp \left(k_{z 2} z\right) & z<-d \\
b_{2} \sinh \left(k_{z 1} z\right) & |z| \leqslant d \\
-b_{1} \exp \left(-k_{z 2} z\right) & z>d
\end{array}, \quad\right. \text { AS } \\
\left\{\begin{array}{ll}
b_{1} \exp \left(k_{z 2} z\right) & z<-d \\
b_{2} \cosh \left(k_{z 1} z\right) & |z| \leqslant d \\
b_{1} \exp \left(-k_{z 2} z\right) & z>d
\end{array}, \quad \mathrm{~S}\right.\end{cases}
\end{aligned}
$$

For the $\rho-\mathrm{IO} / z-\mathrm{PR}$ mixing modes, their electrostatic potentials can be written as

$$
\begin{aligned}
\Phi_{m}^{\rho-\mathrm{IO} / z-\mathrm{PR}}(\mathbf{r}) & =\mathrm{e}^{\mathrm{i} m \varphi} f^{\mathrm{IO}}(\rho) \phi^{\mathrm{PR}}(z), \\
f^{\mathrm{IO}}(\rho) & =\left\{\begin{array}{ll}
A_{1} I_{m}\left(k_{t 1} \rho\right) & \rho \leqslant R \\
A_{2} K_{m}\left(k_{t 2} \rho\right) & \rho>R
\end{array},\right. \\
\phi^{\mathrm{PR}}(z) & = \begin{cases} \begin{cases}B_{1} \exp \left(\mathrm{i} k_{z 2} z\right) & z<-d \\
B_{2} \sin \left(k_{z 1} z\right) & |z| \leqslant d \\
-B_{1} \exp \left(\mathrm{i} k_{z 2} z\right) & z>d \\
B_{1} \exp \left(\mathrm{i} k_{z 2} z\right) & z<-d \\
B_{3} \cos \left(k_{z 1} z\right) & |z| \leqslant d, \\
B_{1} \exp \left(\mathrm{i} k_{z 2} z\right) & z>d\end{cases} \end{cases}
\end{aligned}
$$

In equation (3), $J_{m}(x)$ and $Y_{m}(x)$ are the Bessel and Neumann functions of $m$-order, respectively. In equation (4), $K_{m}(x)$ and $I_{m}(x)$ are the first- and second-kind modified Bessel functions of the order $m$, respectively. Here $a_{i}\left(A_{i}\right)$ and $b_{i}\left(B_{i}\right)$ are coupling coefficients of phonon modes determined by additional boundary conditions (BCs). Due to the complexity of the coupling coefficients, they are not displayed completely here. Only some important coupling coefficients $b_{2}, B_{2}$ and $B_{3}$ are given in the Appendix [because the three coefficients appear in equations (10)-(15) and Fröhlich electron-phonon interaction Hamiltonian (19)]. The other coefficients can be referred to in the Appendixes of [5] and [41]. The symbols "AS" and "S" in equations (3) and (4) correspond to the antisymmetrical solution and symmetrical solution, respectively. This treatment completely satisfies the symmetry demand of the phonon potential in $z$-direction. According to the relationship of Loudon's uniaxial crystal model [31] and the Laplace equation (2) in the areas of the inner and outer $\mathrm{QD}$, the dependent relations of the phonon wave-numbers $k_{u v}(u=t, z ; v=1,2)$ can be chosen as

$$
\begin{aligned}
\sqrt{\epsilon_{t i}(\omega)} k_{t i} \pm \sqrt{\epsilon_{z i}(\omega)} k_{z i} & =0 \\
\sqrt{\epsilon_{z 1}(\omega)} k_{z 1} \pm \sqrt{\epsilon_{z 2}(\omega)} k_{z 2} & =0
\end{aligned}
$$

It should be noted that the subscripts 1 and 2 in equations (3), (44) and (5) correspond to the GaN material and vacuum dielectric environment, respectively. The reasonableness of equation (5) lies in the fact that the present theories in wurtzite Q0D QDs can naturally reduce to the corresponding results of Q1D QWR and Q2D QW structures well known under a certain condition, which will be discussed in detail hereinafter.

Next, we deduce the dispersive equations of the IO-PR mixing phonon modes. For the $\rho-\mathrm{IO} / z-\mathrm{PR}$ mixing modes, using the continuity BCs of the potential functions and the electric displacement vector at the axial interfaces $z= \pm d$ and the radial interface $\rho=R$, one can get the following two equations [5, 9], i.e.

$$
k_{z 1}=n \pi / 2 d, \quad n= \pm 1, \pm 2, \ldots
$$


and

$\epsilon_{t, 1} k_{t 1} K_{m}\left(k_{t 2} R\right)\left[I_{m-1}\left(k_{t 1} R\right)+I_{m+1}\left(k_{t 1} R\right)\right]=-\epsilon_{t 2} k_{t 2} I_{m}\left(k_{t 1} R\right)\left[K_{m-1}\left(k_{t 2} R\right)+K_{m+1}\left(k_{t 2} R\right)\right]$.

In equation (6), $n$ taking even (odd) number corresponds to the symmetric (antisymmetric) $\rho-\mathrm{IO} / z-\mathrm{PR}$ mixing phonon modes. Connecting equations (15), (6) and (7), the dispersive frequencies of $\rho-\mathrm{IO} / z-\mathrm{PR}$ mixing modes can be worked out. In the same way, via the continuity BCs of the potential functions and the electric displacement vector at the axial interfaces $z= \pm d$ and the radial interface $\rho=R$, one can obtain two other dispersive equations (8) and (9) for the $z-\mathrm{IO} / \rho-\mathrm{PR}$ mixing modes [4, 10]. They are given by

$$
k_{z 1}= \begin{cases}\arctan \mathrm{h} \sqrt{-\epsilon_{z 2} / \epsilon_{z 1}} / d, & \mathrm{~S} \\ \arctan \mathrm{h} \sqrt{-\epsilon_{z 1} / \epsilon_{z 2}} / d, & \mathrm{AS}\end{cases}
$$

and

$$
\begin{aligned}
& \left\{k_{t 2} \epsilon_{t, 2} J_{m}\left(k_{t 1} R\right)\left[J_{m-1}\left(k_{t 2} R\right)-J_{m+1}\left(k_{t 2} R\right)\right]\right. \\
& \left.\quad+k_{t 1} \epsilon_{t, 1} J_{m}\left(k_{t 2} R\right)\left[J_{m+1}\left(k_{t 1} R\right)-J_{m-1}\left(k_{t 1} R\right)\right]\right\} Y_{m}\left(k_{t 2} L\right) \\
& =\quad J_{m}\left(k_{t 2} L\right)\left\{k_{t 1} \epsilon_{t, 1}\left[J_{m+1}\left(k_{t 1} R\right)-J_{m-1}\left(k_{t 1} R\right)\right] Y_{m}\left(k_{t 2} R\right)\right. \\
& \left.\quad+k_{t 2} \epsilon_{t, 2}\left[Y_{m+1}\left(k_{t 2} R\right)-Y_{m-1}\left(k_{t 2} R\right)\right] J_{m}\left(k_{t 1} R\right)\right\} .
\end{aligned}
$$

In the same way, "S" and "AS" in equation (18) also denote the symmetric and antisymmetric $z-\mathrm{IO} / \rho-\mathrm{PR}$ mixing modes. In equation (9), $L$ is the maximum radial size of the nonpolar dielectric environment (in general, $L \gg R$ ). The dispersive frequencies and properties of the $z-\mathrm{IO} / \rho-\mathrm{PR}$ mixing modes in GaN QDs can be completely obtained by numerically solving the equations (8) and (9).

\subsection{Free phonon fields and Fröhlich electron-phonon interaction Hamiltonians}

To obtain the expressions for the Hamiltonian of the free phonon field and electron-phonon interaction Hamiltonian, we first institute the orthogonal relationships of polarization vector of IO-PR mixing phonon modes. Using the formula $\mathbf{P}=(1-\epsilon) / 4 \pi \nabla \Phi(\mathbf{r})$, and via equation (3), we obtain the orthogonal relations of polarization vector for $z-\mathrm{IO} / \rho-\mathrm{PR}$ mixing modes, i.e.

$$
\begin{aligned}
\int \mathbf{P}_{\mathrm{AS}, m^{\prime}}^{z-\mathrm{IO}^{\prime} \rho-\mathrm{PR}^{*}} \cdot \mathbf{P}_{\mathrm{AS}, m}^{z-\mathrm{IO} / \rho-\mathrm{PR}^{3} \mathbf{r}} & \\
= & \frac{\left|A_{0}\right|^{2}}{16 \pi} \int \rho \mathrm{d} \rho \mathrm{d} z\left\{b_{2}^{2} \sinh \left(k_{z 1} z\right)^{2}\left(1-\epsilon_{t 1}\right)^{2} k_{t 1}^{2}\left[J_{m-1}^{2}\left(k_{t 1} \rho\right)+J_{m+1}^{2}\left(k_{t 1} \rho\right)\right]\right. \\
& \left.\quad+2 J_{m}^{2}\left(k_{t 1} \rho\right)\left(1-\epsilon_{z 1}\right)^{2} k_{z 1}^{2} b_{2}^{2} \cosh \left(k_{z 1} z\right)^{2}\right\} \delta_{m^{\prime} m}
\end{aligned}
$$

and

$$
\begin{aligned}
& \int \mathbf{P}_{\mathrm{S}, m^{\prime}}^{z-\mathrm{IO}^{\prime} / \rho-\mathrm{PR}^{*}} \cdot \mathbf{P}_{\mathrm{S}, m}^{z-\mathrm{IO} / \rho-\mathrm{PR}^{3} \mathbf{r}} \\
& =\frac{\left|A_{0}\right|^{2}}{16 \pi} \int \rho \mathrm{d} \rho \mathrm{d} z\left\{b_{2}^{2} \cosh \left(k_{z 1} z\right)^{2}\left(1-\epsilon_{t 1}\right)^{2} k_{t 1}^{2}\left[J_{m-1}^{2}\left(k_{t 1} \rho\right)+J_{m+1}^{2}\left(k_{t 1} \rho\right)\right]\right. \\
& \left.\quad+2 J_{m}^{2}\left(k_{t 1} \rho\right)\left(1-\epsilon_{z 1}\right)^{2} k_{z 1}^{2} b_{2}^{2} \sinh \left(k_{z 1} z\right)^{2}\right\} \delta_{m^{\prime} m}
\end{aligned}
$$

for the antisymmetrical and symmetrical $z-\mathrm{IO} / \rho-\mathrm{PR}$ mixing phonon modes, respectively. Based on equation (4), the orthogonal relations of polarization vectors for symmetrical and antisymmetrical $\rho-\mathrm{IO} / z-\mathrm{PR}$ mixing modes are unified as

$$
\begin{aligned}
\int & \mathbf{P}_{\mathrm{AS} / \mathrm{S}, m^{\prime}}^{\rho-\mathrm{IO} / z-\mathrm{PR}^{*}} \cdot \mathbf{P}_{\mathrm{AS} / \mathrm{S}, m}^{\rho-\mathrm{IO} / z-\mathrm{PR}^{3}} \mathrm{~d}^{3} \mathbf{r} \\
= & \frac{\left|\mathcal{A}_{0}\right|^{2}}{16 \pi} \int \rho \mathrm{d} \rho \mathrm{d} z\left\{\left|B_{2} \cos \left(k_{z 1} z\right)+B_{3} \sin \left(k_{z 1} z\right)\right|^{2}\left(1-\epsilon_{t 1}\right)^{2} k_{t 1}^{2} K_{m}^{2}\left(k_{t 2} R\right)\left[I_{m-1}^{2}\left(k_{t 1} \rho\right)+I_{m+1}^{2}\left(k_{t 1} \rho\right)\right]\right. \\
& \left.\quad+2 K_{m}^{2}\left(k_{t 2} R\right) I_{m}^{2}\left(k_{t 1} \rho\right)\left(1-\epsilon_{z 1}\right)^{2} k_{z 1}^{2}\left|B_{2} \sin \left(k_{z 1} z\right)+B_{3} \cos \left(k_{z 1} z\right)\right|^{2}\right\} \delta_{m^{\prime} m}
\end{aligned}
$$


When deducing the above orthogonal relations of polarization vectors (10)-(12), only the region inside the cylindrical QDs is considered, and the region outside the QDs is neglected because the polarization vectors in this region is null due to $\epsilon_{d}=1$. Furthermore, it is also observed from these equations that only the azimuthal quantum number $m$ is a good quantum number originating from the symmetry of cylindrical QDs, which is obviously different from the situations of QWs and QWRs systems [2 5, 9, 10, 29]. Choosing suitable normalization constants $A_{0}\left(\mathcal{A}_{0}\right)$, i.e.,

$$
\begin{aligned}
\left|A_{0}\right|^{2}= & \left\{\frac { 1 } { 2 \omega ^ { 2 } } \int \rho \mathrm { d } \rho \mathrm { d } z \left\{b_{2}^{2} \sinh ^{2}\left(k_{z 1} z\right) \bar{\epsilon}_{t 1} k_{t 1}^{2}\left[J_{m-1}^{2}\left(k_{t 1} \rho\right)+J_{m+1}^{2}\left(k_{t 1} \rho\right)\right]\right.\right. \\
& \left.\left.+2 J_{m}^{2}\left(k_{t 1} \rho\right) \bar{\epsilon}_{z 1} k_{z 1}^{2} b_{2}^{2} \cosh ^{2}\left(k_{z 1} z\right)\right\}\right\}^{-1}
\end{aligned}
$$

for antisymmetrical $z-\mathrm{IO} / \rho-\mathrm{PR}$ mixing modes,

$$
\begin{aligned}
\left|A_{0}\right|^{2}= & \left\{\frac { 1 } { 2 \omega ^ { 2 } } \int \rho \mathrm { d } \rho \mathrm { d } z \left\{b_{2}^{2} \cosh ^{2}\left(k_{z 1} z\right) \bar{\epsilon}_{t 1} k_{t 1}^{2}\left[J_{m-1}^{2}\left(k_{t 1} \rho\right)+J_{m+1}^{2}\left(k_{t 1} \rho\right)\right]\right.\right. \\
& \left.\left.+2 J_{m}^{2}\left(k_{t 1} \rho\right) \bar{\epsilon}_{z 1} k_{z 1}^{2} b_{2}^{2} \sinh ^{2}\left(k_{z 1} z\right)\right\}\right\}^{-1}
\end{aligned}
$$

for symmetrical $z-\mathrm{IO} / \rho-\mathrm{PR}$ mixing modes, and

$$
\begin{aligned}
\left|\mathcal{A}_{0}\right|^{2}= & \left\{\frac { 1 } { 2 \omega ^ { 2 } } \int \rho \mathrm { d } \rho \mathrm { d } z \left\{\left|B_{2} \cos \left(k_{z 1} z\right)+B_{3} \sin \left(k_{z 1} z\right)\right|^{2} \bar{\epsilon}_{t 1} k_{t 1}^{2} K_{m}^{2}\left(k_{t 2} R\right)\left[I_{m-1}^{2}\left(k_{t 1} \rho\right)+I_{m+1}^{2}\left(k_{t 1} \rho\right)\right]\right.\right. \\
& \left.\left.+2 K_{m}^{2}\left(k_{t 2} R\right) I_{m}^{2}\left(k_{t 1} \rho\right) \bar{\epsilon}_{z 1} k_{z 1}^{2}\left|B_{2} \sin \left(k_{z 1} z\right)+B_{3} \cos \left(k_{z 1} z\right)\right|^{2}\right\}\right\}^{-1}
\end{aligned}
$$

for the symmetrical and antisymmetrical $\rho-\mathrm{IO} / z-\mathrm{PR}$ mixing phonon modes, the polarization vectors can be treated as orthogonal and complete sets, which can be used to express the free phonon field $H_{\mathrm{IO}-\mathrm{PR}}$ of IO-PR mixing modes and the Hamiltonians $H_{\mathrm{e}-\mathrm{ph}}$ of electron-(IO-PR) mixing phonons interactions. In equation (13)-(15), $\bar{\epsilon}_{v 1}(v=t, z)$ is the effective dielectric function of the GaN material, which is defined as

$$
\bar{\epsilon}_{v}=\left(\frac{1}{\epsilon_{v}-\epsilon_{v 0}}-\frac{1}{\epsilon_{v}-\epsilon_{v \infty}}\right)^{-1}, \quad v=t, z .
$$

Using the orthogonal relations (10)-(12) and following the quantization steps similar to those in [2 [5], one can obtain the free phonon Hamiltonian operators for the IO-PR mixing phonons, i.e.

$$
H_{\mathrm{IO}-\mathrm{PR}}=\sum_{m, k_{z 1}} \hbar \omega\left[b_{m}^{\dagger}\left(k_{z 1}\right) b_{m}\left(k_{z 1}\right)+\frac{1}{2}\right]
$$

where $b_{m}^{\dagger}\left(k_{z 1}\right)$ and $b_{m}\left(k_{z 1}\right)$ are creation and annihilation operators for the IO-PR mixing phonons of $m$ th modes. They satisfy the commutative rules for bosons:

$$
\begin{aligned}
& {\left[b_{m}\left(k_{z 1}\right), b_{m^{\prime}}^{\dagger}\left(k_{z 1}\right)\right]=\delta_{m^{\prime} m}} \\
& {\left[b_{m}\left(k_{z 1}\right), b_{m^{\prime}}\left(k_{z 1}\right)\right]=\left[b_{m}^{\dagger}\left(k_{z 1}\right), b_{m^{\prime}}^{\dagger}\left(k_{z 1}\right)\right]=0 .}
\end{aligned}
$$

The interaction Hamiltonians of electron with the IO-PR phonon fields is read as $H_{\mathrm{e}-\mathrm{ph}}=$ $-\mathrm{e} \Phi^{\mathrm{IO}-\mathrm{PR}}(\mathbf{r})$. Expanding $\Phi^{\mathrm{IO}-\mathrm{PR}}(\mathbf{r})$ in terms of the normal modes, and after some trivial algorithms, we get the electron-(IO-PR)phonons interaction Hamiltonians as

$$
\begin{aligned}
H_{\mathrm{e}-\mathrm{ph}} & =-\mathrm{e} \sum_{m, k_{z 1}}\left(\frac{\hbar}{8 \pi \omega}\right)^{1 / 2}\left[b_{m}^{\dagger}\left(k_{z 1}\right)+b_{m}\left(k_{z 1}\right)\right] \Phi_{m}^{\mathrm{IO}-\mathrm{PR}}(\mathbf{r}) \\
& =-\sum_{m, k_{z 1}} \Gamma_{m, k_{z 1}}^{\mathrm{IO}-\mathrm{PR}}(\rho) \Gamma_{m, k_{z 1}}^{\mathrm{IO}-\mathrm{PR}}(z)\left[b_{m}\left(k_{z 1}\right) \mathrm{e}^{\mathrm{i} m \varphi}+\text { H.c. }\right]
\end{aligned}
$$


where $\Gamma_{m, k_{z 1}}^{\mathrm{IO}-\mathrm{PR}}(\rho)$ and $\Gamma_{m, k_{z 1}}^{\mathrm{IO}-\mathrm{PR}}(z)$ are the coupling functions defined as

$$
\begin{aligned}
\Gamma_{m, k_{z 1}}^{\mathrm{IO}-\mathrm{PR}}(\rho) & =\sqrt{\left|N_{m}\left(k_{z 1}\right)\right|} f^{\mathrm{IO}, \mathrm{PR}}(\rho), \\
\Gamma_{m, k_{z 1}}^{\mathrm{IO}-\mathrm{PR}}(z) & =\sqrt{\left|N_{m}\left(k_{z 1}\right)\right|} \phi^{\mathrm{IO}, \mathrm{PR}}(z),
\end{aligned}
$$

with

$$
\left|N_{m}\left(k_{z 1}\right)\right|=\sqrt{\frac{\hbar \mathrm{e}^{2}}{\omega}}\left|A_{0}\right| .
$$

In equation (20), the functions $f^{\mathrm{SO}, \mathrm{PR}}(\rho)$ and $\phi^{\mathrm{SO}, \mathrm{PR}}(z)$ are defined in equation (3) and equation (4).

\section{Numerical results and discussion}

In the present section, we will numerically discuss the quantum size effect on the dispersive properties of IO-PR mixing phonon modes in a wurtzite GaN cylindrical QD. The physical parameters of the materials used in our calculations are listed in table 1.

Table 1. Zone-center energies (in $\mathrm{cm}^{-1}$ ) of polar optical phonons, dielectric constants of wurtzite GaN material and dielectric matrix $[3,4]$.

\begin{tabular}{ccccccc}
\hline $\mathrm{A}_{1}(\mathrm{TO})$ & $\mathrm{E}_{1}(\mathrm{TO})$ & $\mathrm{A}_{1}(\mathrm{LO})$ & $\mathrm{E}_{1}(\mathrm{LO})$ & $\epsilon_{\infty}$ & $\epsilon_{0}$ & $\epsilon_{d}$ \\
\hline 532 & 559 & 734 & 741 & 5.35 & 9.2 & 1 \\
\hline
\end{tabular}

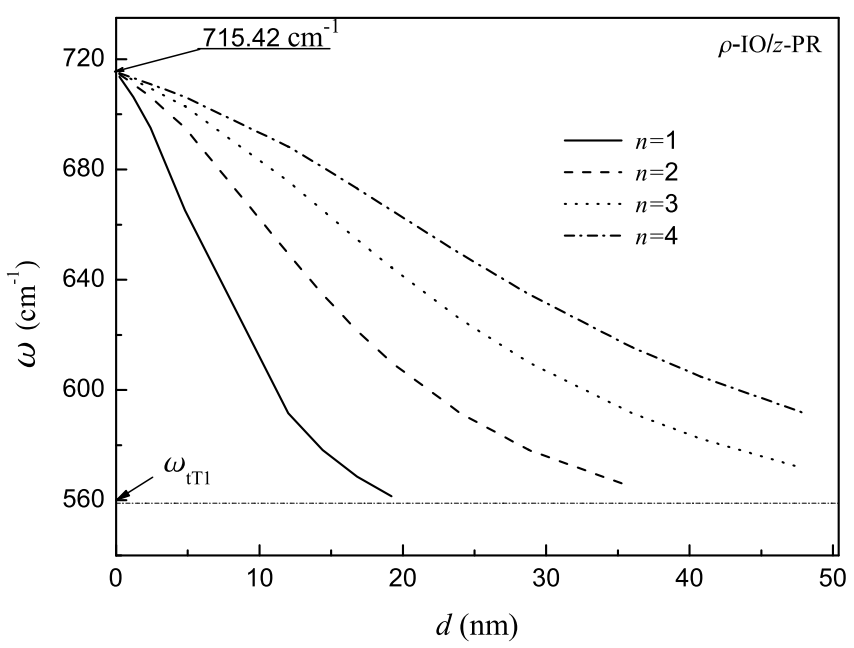

Figure 1. Dispersive frequencies $\omega$ of the first four branches of $\rho$-IO/z-PR mixing modes $(n=$ $1,2,3,4)$ as a function of the half-height $d$ of the wurtzite GaN cylindrical QDs when the radius $R=4.8 \mathrm{~nm}$ and the azimuthal quantum number $m=0$.

Let us first investigate the dispersive feature of the $\rho$-IO/z-PR mixing phonon modes. In figure 1 , the dispersive frequencies $\omega$ of the $\rho$-IO/z-PR mixing modes as a function of the half-height $d$ of the wurtzite GaN cylindrical QDs are plotted when the radius $R$ takes $4.8 \mathrm{~nm}$ and the azimuthal quantum number $m$ is kept at 0 . Only the first four branches $(n=1,2,3,4)$ of $\rho$-IO $/ z$-PR mixing modes with $m=0$ are depicted. In fact, for a certain half-height $d$ and a given azimuthal quantum 
number $m$, the equations (6) and (7) have infinite solutions for $\omega$, which means that there exists infinite branches of $\rho$-IO/z-PR mixing modes in the GaN QD structures. From the figure, it can be seen that all the phonon branches start with the constant frequency value $715.42 \mathrm{~cm}^{-1}$, and decrease monotonously to the characteristic frequency $\omega_{\mathrm{tT} 1}$ of GaN material with the increase of $d$. The decline of low-order modes ( $n$ is small) is steeper than that of the high-order ones $(n$ is relatively large). Via a detailed analysis, it is found that the frequency value of $715.42 \mathrm{~cm}^{-1}$ is just the root of equation $\epsilon_{t 1}=1$, and this equation also determines the limiting frequencies of phonon modes for quite large wave-numbers in wurtzite QW and QWR structures [4, 5, 9]. This interesting feature needs to be further explained. From a pure viewpoint of mathematics, as $d \rightarrow 0$, the phonon wave-numbers $k_{z 1}$ and $k_{u 2}(u=t, z)$ will approach the infinity via equations (6) and (5). Based on the limiting relations of modified Bessel functions [42], i.e.

$$
\begin{aligned}
\lim _{x \rightarrow \infty} I_{m}(x) & =\mathrm{e}^{x} / \sqrt{2 \pi x} \\
\lim _{x \rightarrow \infty} K_{m}(x) & =\mathrm{e}^{-x} \sqrt{\pi} / \sqrt{2 x}
\end{aligned}
$$

it is easy to prove that, as $k_{t v} \rightarrow \infty(v=1,2)$, equation (7) will degenerate to the form of $\epsilon_{t 1}=1$. This equation just gives the frequency of $715.42 \mathrm{~cm}^{-1}$. From a physical viewpoint, the wave-lengths of mixing phonon modes become very short as $k_{u v} \rightarrow \infty(u=t, z ; v=1,2)$, thus the phonons cannot distinguish planar heterostructure and the curved cylindrical heterostructure [43]. This directly results in the identical limiting frequency of phonon modes in wurtzite QW, QWR and QD systems for very large wave-numbers [4, 5, 9]. On the other hand, we observe that the dispersive curve of $n=1 \rho$-IO/z-PR mode is cut off at about $d=19 \mathrm{~nm}$, and that of $n=2$ mode is cut off at about $d=36 \mathrm{~nm}$. The other two mixing phonon modes with $n=3,4$ are also cut off at two certain $d$. These are the typical "reducing" behaviors of confined phonon modes in wurtzite quantum structures [2-10]. In fact, as $\omega$ is lower than $\omega_{t T 1}$, the sign of $\epsilon_{t 1}(\omega) \epsilon_{z 1}(\omega)$ will become negative, thus the $\rho$ - $\mathrm{IO} / z$-PR mixing phonon modes cannot exist in this situation, and they will reduce to the other phonon modes, such as the half-space modes or quasi-confined modes $[2[5,9,10]$.

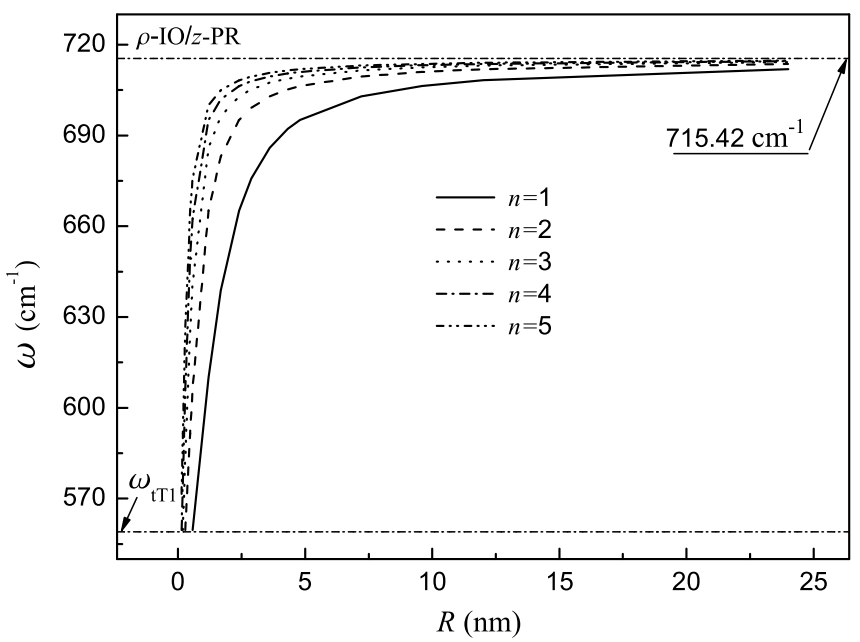

Figure 2. Dispersive frequencies $\omega$ of the first five branches of $\rho-\mathrm{IO} / z$-PR mixing phonon modes as a function of the radius $R$ when $m=0$ and $d=2.4 \mathrm{~nm}$.

The dispersive frequencies $\omega$ of $\rho$-IO/z-PR mixing phonon modes as a function of the radius $R$ of the wurtzite GaN QDs are depicted in figure 2. A certain azimuthal quantum number $(m=0)$ and a certain half-height $(d=2.4 \mathrm{~nm})$ are chosen to plot the figure. For clarity, only the first five 
branches of mixing phonon modes are shown here. It is explicitly seen that the frequency range of the $\rho$-IO/z-PR mixing modes is $\left[715.42 \mathrm{~cm}^{-1}, \omega_{t T 1}\right]$, which is exactly as the case in figure 1 . All the curves are the monotonic and decreasing functions of $R$. The "reducing" behavior of $\rho$-IO $/ z$-PR mixing phonon modes is also observed again in the figure, namely the curves are cut off at $\omega=\omega_{t T 1}$ as the radius $R$ approaches 0 . When $R$ approaches $\infty$, the frequencies of all the $\rho$-IO/z-PR modes converge to the constant frequency value of $715.42 \mathrm{~cm}^{-1}$. In fact, via the limiting relations (22) of modified Bessel functions, the dispersive equation (7) can also degenerate to the form of $\epsilon_{t 1}=1$, thus the limiting frequencies of $\rho$-IO/z-PR mixing phonon modes for very large $R$ also converge to the constant of $715.42 \mathrm{~cm}^{-1}$.

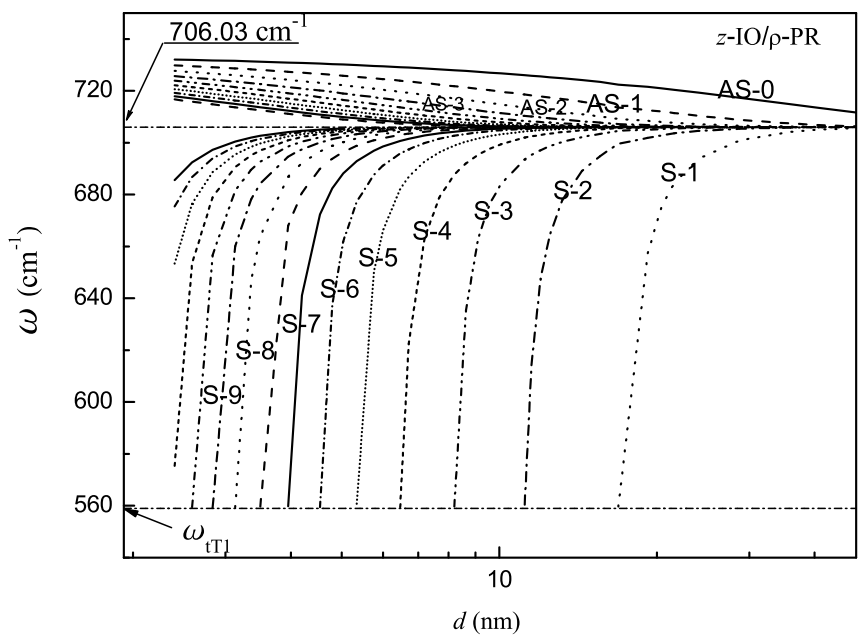

Figure 3. Dispersive curves of the $z-\mathrm{IO} / \rho$-PR mixing modes as a function of $d$ when $R=$ $4.8 \mathrm{~nm}$ and $m=0$. The symbols of $\mathrm{S}-i(\mathrm{AS}-i)$ in the figure denote the $i$-order symmetrical (antisymmetrical) $z$-IO $/ \rho$-PR modes.

The dispersive curves of the $z$-IO $/ \rho$-PR mixing modes as a function of the half-height $d$ of the GaN QDs are plotted in figure 3 when $R$ and $m$ are respectively fixed at $4.8 \mathrm{~nm}$ and 0 . From the figure, it is clearly seen that dispersive curves of $z-\mathrm{IO} / \rho$-PR mixing phonon modes are distributed in the frequency range of $\left[\omega_{t T 1}, \omega_{z L 1}\right]$. This range is divided into two ranges at $\omega=706.03 \mathrm{~cm}^{-1}$. The above range of $\left[706.03 \mathrm{~cm}^{-1}, \omega_{z L 1}\right]$ is the frequency range of the antisymmetrical $z-\mathrm{IO} / \rho$-PR mixing modes, while neither range of $\left[\omega_{t T 1}, 706.03 \mathrm{~cm}^{-1}\right]$ is the frequency range of the symmetrical $z-\mathrm{IO} / \rho$ $\mathrm{PR}$ mixing modes. In the figure, the symmetrical (antisymmetrical) $z$-IO $/ \rho$-PR modes are labeled by $\mathrm{S}-i(\mathrm{AS}-i)$. Based on equations (8) and (9), there are infinite branches of $z$-IO $/ \rho$-PR mixing modes in the symmetrical and antisymmetrical frequency ranges, and only the first fourteen branches of $z$-IO $/ \rho$-PR modes in each frequency range are shown. The index $i$ in symbols of $\mathrm{S} / \mathrm{AS}-i$ denotes the node-number of the electrostatic potential of $z$-IO $/ \rho$-PR modes in $t$-direction. Using this feature, all the symmetrical and antisymmetrical $z-\mathrm{IO} / \rho-\mathrm{PR}$ modes are labeled and distinguished. It is necessary to briefly discuss the origin of the characteristic value of $706.03 \mathrm{~cm}^{-1}$. In fact, the frequency of $706.03 \mathrm{~cm}^{-1}$ is just the solution of equation, $\epsilon_{z 1}=1$. As $d \rightarrow \infty$, the wave-numbers $k_{z 1}$ approach 0 via the relationship (8). In terms of the nature of Bessel function and Neumann function [42]:

$$
\begin{aligned}
& \lim _{x \rightarrow 0} J_{m}(x) \propto \frac{1}{\Gamma(m+1)}\left(\frac{x}{2}\right)^{m}, \\
& \lim _{x \rightarrow 0} Y_{m}(x) \propto\left\{\begin{array}{ll}
-\frac{2}{\pi} \ln (x / 2)^{-m} & m=0 \\
-\frac{\Gamma(m)}{\pi}(x / 2)^{-m} & m>0
\end{array},\right.
\end{aligned}
$$


the dispersive equation (9) will reduce to the form of $\epsilon_{z 1}=1$ as the wave-numbers $k_{t 1}$ and $k_{t 2}$ approach 0 . This distinctly explains the mathematic origin of the frequency values of $706.03 \mathrm{~cm}^{-1}$. The profound physical origin lies in the fact that the $z$-IO $/ \rho$-PR mixing phonon modes of GaN QDs will reduce to the corresponding phonon modes in Q1D GaN QWRs [9, 10] as $d \rightarrow \infty$, which will be analyzed in detail hereinafter. We also notice that, from the figure, the lowest-order $z$-IO $/ \rho$-PR mode in high frequency range is $\mathrm{AS}-0$, but the lowest-order $z$ - $\mathrm{IO} / \rho$-PR mode in low frequency range is $\mathrm{S}-1$. All the symmetrical (antisymmetrical) $z$-IO $/ \rho$-PR modes are the monotonous and incremental (decreasing) function of $R$. By contrast to the symmetrical $z$-IO $/ \rho$-PR modes, the dispersions of antisymmetrical $z$-IO/ $\rho$-PR modes are weaker. Moreover, the symmetrical $z$-IO $/ \rho$-PR modes explicitly indicate the "reducing" behavior of phonon modes in wurtzite confined systems, i.e. they are cut off at the characteristic frequency $\omega_{t T 1}$ of GaN at a series of certain values of $d$.

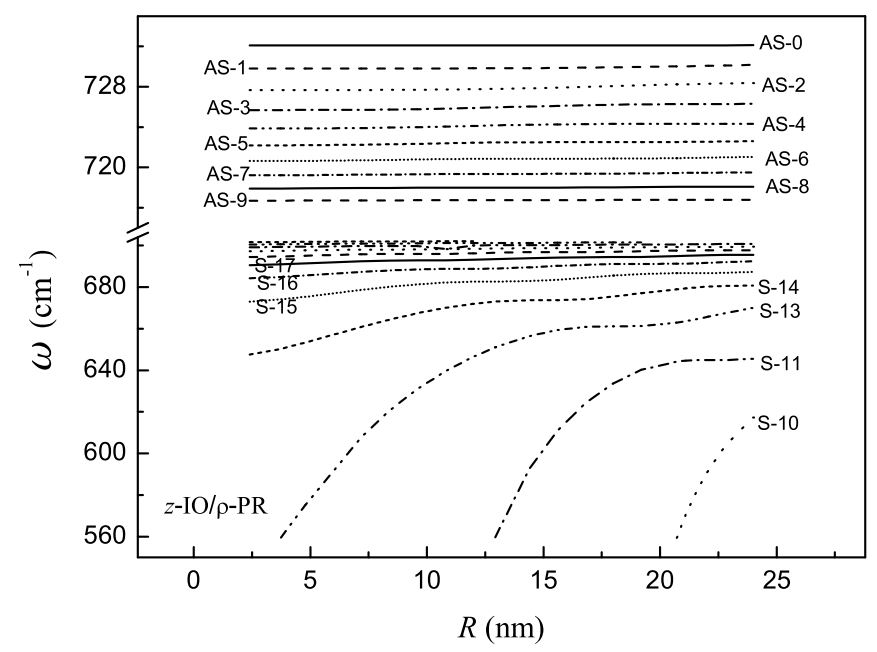

Figure 4. Dispersive frequencies $\omega$ of $z-\mathrm{IO} / \rho$-PR mixing phonon modes as a function of $R$ of the GaN QDs when the azimuthal quantum number $m=0$ and the half-height $d=2.4 \mathrm{~nm}$. The meanings of the symbols $(\mathrm{S} / \mathrm{AS}-i)$ in the figure are the same as those in figure 3

Figure 4 depicts the dispersive frequency $\omega$ of $z-\mathrm{IO} / \rho$-PR mixing phonon modes as a function of the radius $R$ of the GaN QDs. Same as in figure 2, $m=0$ and $d=2.4 \mathrm{~nm}$ are chosen when plotting the figure. The meanings of the symbols $(\mathrm{S} / \mathrm{AS}-i)$ in the figure are the same as those in figure 3 . It is observed that all the curves are located in two frequency ranges, i.e. the higher-frequency range (antisymmetrical $z$-IO/ $\rho$-PR modes) and the lower-frequency range (symmetrical $z$-IO $/ \rho$-PR modes), which is quite similar to the case in figure 3. The symmetrical $z$-IO $/ \rho$-PR modes are more dispersive than the antisymmetrical modes. Moreover, the dispersions of the lower-order $(i$ of S- $i$ is small) symmetrical $z$-IO/ $\rho$-PR modes are more explicit than those of the higher-order modes. With the increase of $R$, the frequencies of antisymmetrical $z$-IO/ $\rho$-PR modes nearly remain unchanged. As stated in figure 3 , the $i$ of S/AS- $i$ represents the node-numbers of phonon electrostatic potentials in $t$-direction. From the figure, we find that, as $R<24 \mathrm{~nm}$, only the symmetrical $z$-IO $/ \rho$-PR modes with the order higher than $10(i \geqslant 10)$ appear. However, all the antisymmetrical $z$-IO $/ \rho$-PR modes (from zero-order to infinity order) can be observed (only the first ten branches antisymmetrical modes are shown here).

Now let us briefly discuss the relation and difference between the present phonon modes theories of wurtzite Q0D QDs and those of wurtzite Q1D (Q2D) QWR (QW) structures [2 5, 9, 10]. From a purely mathematical viewpoint, as the half-height of GaN QDs $d \rightarrow \infty$, the wave-numbers $k_{z 1}$ of IO-PR mixing phonon modes will become continuous via equations (6) or (8). Under this condition, 
based on equation (5) one can get the relations:

$$
\begin{aligned}
k_{z 1} & =k_{z 2}=k_{z}, \\
k_{t i} & =\sqrt{\epsilon_{z i}(\omega) / \epsilon_{t i}(\omega)} k_{z} .
\end{aligned}
$$

Substituting the two conditions of equation (24) into the equations (7) or (9), equation (7) [equation (9)] will reduce to the form of dispersive equation which is exactly the same as the dispersive equation of IO (PR) phonon modes in Q1D wurtzite QWRs [9, 10]. This distinctly explains the fact, from a mathematic viewpoint, that the $\rho-\mathrm{IO} / z-\mathrm{PR}(z-\mathrm{IO} / \rho-\mathrm{PR})$ mixing phonon modes in Q0D wurtzite QDs reduce to the IO (PR) phonon modes in Q1D wurtzite QWRs under the condition of $d \rightarrow \infty$. From a viewpoint of physics, as the height $d$ approaches $\infty$ and the radius $R$ is kept at a finite value, the physical model of the wurtzite Q0D cylindrical QD structures will naturally reduce to the wurtzite Q1D cylindrical QWR structures. This further proves the correctness and reliability of the phonon modes theories in Q0D wurtzite QD systems established in the present work.

On the other hand, as the height $2 d$ takes a certain value and the radius $R$ approaches infinity, via the limiting relations of Bessel function and Neumann function [42]:

$$
\begin{aligned}
\lim _{x \rightarrow \infty} J_{m}(x) & =\sqrt{\frac{2}{\pi x}} \cos \left[x-\left(m+\frac{1}{2}\right)\right] \frac{\pi}{2}, \\
\lim _{x \rightarrow \infty} Y_{m}(x) & =\sqrt{\frac{2}{\pi x}} \sin \left[x-\left(m+\frac{1}{2}\right)\right] \frac{\pi}{2},
\end{aligned}
$$

the wave-number $k_{t i}(i=1,2)$ will become continuous based on equation (9). From equations (5), one can get the relations:

$$
\begin{aligned}
k_{t 1} & =k_{t 2}=k_{t}, \\
k_{z i} & =\sqrt{\epsilon_{t i}(\omega) / \epsilon_{z i}(\omega)} k_{t} .
\end{aligned}
$$

Thus the equation (8) reduces to the dispersive equation of IO phonon modes in Q2D wurtzite QWs. This mathematical result clearly illustrates that, when $R \rightarrow \infty$, the $z$-IO/ $\rho$-PR mixing modes will reduce to the IO modes in Q2D wurtzite QWs [3, 4]. In the same way, based on the equations (7) and (22), it can be easily proven that the $\rho$ - $\mathrm{IO} / z$-PR mixing modes of wurtzite QDs will reduce to the PR modes of Q2D wurtzite QW structures [5] as $R$ approaches $\infty$. These are also natural results because the cylindrical QDs will degenerate into Q2D QWs when the radius of the QDs approaches $\infty$.

Finally, we should point out that, due to the effective limit of DCM theories [44 46], the present theoretical schemes and results are meaningful only as the height $2 d$ and radius $R$ of the QD are not very small (such as $2 d$ and $R$ are over $4 \mathrm{~nm}$, i.e. ten GaN monolayers). In addition, the analytical expressions of Fröhlich interactions between electron with IO-PR mixing phonon modes obtained in the present paper are very useful in investigating the polaronic effect on the physical properties of the commonly used nitride-based devices, such as LEDs and LDs based in the Q0D wurtzite QD systems [1].

\section{Conclusions}

In conclusion, important polar optical phonon modes, i.e. the IO-PR mixing modes in a wurtzite cylindrical QD system have been investigated in the present work. The analytical phonon states are obtained. It is found that there exist two types of IO-PR mixing phonon modes in wurtzite cylindrical QD structures, namely the $\rho$-IO/z-PR mixing modes and the $z$-IO $/ \rho$-PR mixing modes. Each IO-PR mixing phonon mode also has two forms. One is symmetrical, and the other one is antisymmetrical. Based on the method of field quantization, the Fröhlich Hamiltonian of electron(IO-PR) mixing phonons interactions is given. The orthogonal relations of polarization vectors 
for these IO-PR mixing modes are derived. Numerical calculations on a wurtzite GaN cylindrical QD are performed. The quantum size effect on the dispersive properties of the IO-PR mixing phonon modes are emphasized in the calculations. The results reveal that both the sizes of radialdirection $R$ and the axial-direction $d$ have a great effect on the dispersive frequencies of the IO-PR mixing phonon modes. The limiting features of dispersive curves for these mixing phonon modes are analyzed in depth from the mathematical and physical viewpoints. The "reducing" behavior of phonon modes in wurtzite quantum confined structures has been explicitly observed. We also find that, as the height or the radius of the Q0D wurtzite cylindrical QDs approaches infinity, both types of the IO-PR mixing modes (i.e. the $\rho$-IO/z-PR modes and the $z$-IO $/ \rho$-PR modes) will reduce to the IO modes or PR modes in Q2D wurtzite QW and Q1D QWR structures. These reducing behaviors of IO-PR mixing modes in wurtzite QDs have been profoundly analyzed both from the viewpoint of physics and mathematics. This shows that the theories of mixing phonon modes in wurtzite Q0D QDs established in the present paper are consistent with those in wurtzite Q2D QW and Q1D QWR systems [2-5, 9, 10]. Therefore, the present theories and numerical results turn out to be correct and reliable. We hope that the present work will stimulate further theoretical and experimental investigations into lattice dynamical properties, as well as the device applications based on the Q0D wurtzite QD systems.

\section{Acknowledgements}

The author acknowledges the kind help and valuable discussions of Prof. J.J. Shi (Peking University). This work was supported by Science and Technology Project of Advanced Academy of Guangzhou City under Grant No. 2060, P. R. China.

\section{Appendix}

The coupling coefficients $b_{2}$ for the symmetrical and antisymmetrical $z$-IO/ $\rho$-PR mixing phonon modes are respectively defined as

$$
b_{2}=\mathrm{e}^{-k_{z 2} d} / \cosh \left(k_{z 1} d\right)
$$

and

$$
b_{2}=-\mathrm{e}^{-k_{z 2} d} / \sinh \left(k_{z 1} d\right) .
$$

The coupling coefficients $B_{2}$ and $B_{3}$ of the symmetrical and antisymmetrical $\rho$-IO/z-PR mixing phonon modes are complex quantities. Thus, they can be written as

$$
B_{i}=B_{\mathrm{R} i}+\mathrm{i} B_{\mathrm{I} i}
$$

where the real quantities $B_{\mathrm{R} i}$ and $B_{\mathrm{I} i}$ denote the real part and the imaginary part of $B_{i}$, respectively. The coupling coefficients $B_{2}$ are given by

$$
B_{\mathrm{R} 2}=2 k_{z 2} \epsilon_{z 2} \cos \left(k_{z 1} d\right) \sin ^{2}\left(k_{z 1} d\right)\left(k_{z 2}^{2} \epsilon_{z 2}^{2}-k_{z 1}^{2} \epsilon_{z 1}^{2}\right) / \mathcal{D},
$$

and

$$
B_{\mathrm{I} 2}=-2 k_{z 1} k_{z 2}^{2} \epsilon_{z 2}^{2} \epsilon_{z 1} \cos \left(2 k_{z 1} d\right) \sin \left(k_{z 1} d\right) / \mathcal{D} .
$$

The coefficients $B_{3}$ are given by

$$
B_{\mathrm{R} 3}=k_{z 2} \epsilon_{z 2} \sin \left(k_{z 1} d\right)\left[k_{z 2}^{2} \epsilon_{z 2}^{2}-k_{z 1}^{2} \epsilon_{z 1}^{2}+\cos \left(2 k_{z 1} d\right)\left(k_{z 2}^{2} \epsilon_{z 2}^{2}+k_{z 1}^{2} \epsilon_{z 1}^{2}\right)\right] / \mathcal{D},
$$

and

$$
B_{\mathrm{I} 3}=0 .
$$

In equations (30)-(33), $\mathcal{D}$ is defined as

$$
\mathcal{D}=k_{z 1} k_{z 2} \epsilon_{z 1} \epsilon_{z 2}\left[\sin \left(k_{z 1} d\right)-\sin \left(3 k_{z 1} d\right)\right]\left[k_{z 1} \epsilon_{z 1} \cos \left(k_{z 2} d\right) \sin \left(k_{z 1} d\right)-k_{z 2} \epsilon_{z 2} \cos \left(k_{z 1} d\right) \sin \left(k_{z 2} d\right)\right]
$$




\section{References}

1. Nakamura S., Chichibu S.F., Introduction to Nitride Semiconductor Blue Lasers and Light Emitting Diodes, 2000, Taylor and Francis, London.

2. Komirenko S.M., Kim K.W., Stroscio M.A., Dutta M., Phys. Rev. B, 1999, 59, 5013.

3. Gleize J., renucci M.A., Frandon J., Demangeot F., Phys. Rev. B, 1999, 60, 15985.

4. Shi J.J., Phys. Rev. B, 2003, 68, 165335.

5. Zhang L., Shi J.J., Commun. Theor. Phys., 2006, 45, 935.

6. Tan L.T., Martin R.W., O’Donnell K.P., Watson I.M., Appl. Phys. Lett., 2006, 89, 101910.

7. Smith M., Lin J.Y., Jiang H.X., Khan A., Chen Q., Salvador A., Botchkarev A., Kim W., Morkoc H., Appl. Phys. Lett., 1997, 70, 2882.

8. Graham D.M., Dawson P., Godfrey M., Kappers M.J., Humphreys C.J., Appl. Phys. Lett., 2006, 89, 211901.

9. Zhang L., Shi J.J., Tansley T.L., Phys. Rev. B, 2005, 71, 245324.

10. Zhang L., Liao J.S., Superlatt. Microstruc., 2008, 43, 28.

11. Liu H.L., Chen C.C., Chia C.T., Yeh C.C., Chen C.H., Yu M.Y., Keller S., DenBaars S.P., Chem. Phys. Lett., 2001, 345, 245.

12. Dhara S., Chandra S., Mangamma G., Kalavathi S., Shankar P., Hsu C.W., Kuo C.C., Chen L.C., Chen K.H., Sriram K.K., Appl. Phys. Lett., 2007, 90, 213104.

13. Hsiao C-L., Tu L-W., Chi T-W., Chen M., Young T-F., Chia C-T., Chang Y-M., Appl. Phys. Lett., 2007, 90, 043102 .

14. Zhang X.B., Taliercio T., Kolliakos S., Lefebvre P., J. Phys.: Condens. Matter, 2001, 13, 7053.

15. Makhanets O.M., Voitsekhivska O.M., Gryschyk A.M., Condensed Matter Physics, 2006, 9, 719.

16. Tkach N.V., Zharkoy V.P., Fizika i Technika Poluprovodnikov, 1999, 33, 598.

17. Jarjour A.F., Oliver R.A., Tahraoui A., Kappers M.J., Humphreys C.J., Taylor R.A., Phys. Rev. Lett., 2007, 99, 197403.

18. Jarjoura A.F., Oliverb R.A., Tahraouic A., Kappersb M.J., Taylora R.A., Humphreysb C.J., Superlatt. Microstru., 2008, 43, 431.

19. Garro N., Cros A., Llorens J.M., Garcia-Cristobal A., Cantarero A.C., Gogneau, Sarigiannidou N.E., Monroy E., Daudin B., Phys. Rev. B, 2006, 74, 075305.

20. Xu T., Zhou L., Wang Y., Özcan A.S., Ludwig K.F., Smith D.J., Moustakas T.D., J. Appl. Phys., 2007, 102, 073517.

21. Doyennette L., Vardi A., Guillot F., Nevou L., Tchernycheva M., Lupu A., Colombelli R., Bahir G., Monroy E., Julien F.H., Superlatt. Microstru., 2006, 40, 262.

22. Nkaoka T., Kako S., Arakawa Y., Tarucha S., Appl. Phys. Lett., 2007, 90, 162109.

23. Jun-jie Shi, Zi-zhao Gan, J. Appl. Phys., 2003, 94, 407.

24. Shi J.J., Tansley T.L., Solid State Commun., 2006, 138, 26.

25. Liu L., Li J., Xiong G., Physica E, 2005, 25, 466.

26. Wang C.X., Xiong G.G., Microelectronics Journal, 2006, 37, 847.

27. Xia C.X., Jiang Fengchn, Wei S.Y., Current Appl. Phys., 2008, 8, 153.

28. Fonoberov V.A., Balandin A.A., Phys. Rev. B, 2004, 70, 233205.

29. Chassaing P.M., Demangeot F., Paillard V., Zwick A., Combe N., Pages C., Kahn M.L., Maisonnat A., Chaudret B., Phys. Rev. B, 2008, 77, 153306.

30. Zhang L., Shi J.J., Xie H.J., Solid State Communications, 2006, 140, 549.

31. Loudon R., Adv. Phys., 1964, 13, 423.

32. Paula A.M. de, Barbosa L.C., Cruz C.H.B., O.L.Alves, Sanjurjo J.A., Cesar C.L., Appl. Phys. Lett., 1996, 69, 357; Supperlattices and Microstructures, 1998, 23, 1103.

33. Hwang Y-N., Park S-H., Phys. Rev. B, 1999, 59, 7258.

34. Baranov A.V., Rakovich Y.P., Donegan J.F., Perova T.S., Moore R.A., Talapin D.V., Rogach A.L., Masumoto Y., Nabiev I., Phys. Rev. B, 2003, 68, 165306.

35. Dzhagan V.M., Valakh M.Y., Raevskaya A.E., Stroyuk A.L. Kuchmiy S.Y., Zahn D.R.T., Nanotechnology, 2008, 19, 305707.

36. Lange H., Artemyev M., Woggon U., Thomsen C., Nanotechnology, 2009, 20, 045705.

37. Comas F., Studart N., Marques G.E., Solid State Commun., 2004, 130, 477; Comas F., Odriazola A., Phys. Stat. Sol. (b), 2005, 242, 1267.

38. Vasilevskiy M.I., Phys. Rev. B, 2002, 66, 195326.

39. Kanyinda-Malu C., la Cruz R.M.de, Phys. Rev. B, 1999, 59, 1621.

40. Yamanaka T., Alexson D., Stroscio M.A., Dutta M., Petroff P., Brown J., Speck J., J. Appl. Phys., 
2008, 104, 093512.

41. Zhang L., Shi J.J., Centr. Eur. J. Phys., 2007, 5, 324.

42. Xi D.P., Bessel functions. China Higher Education Press (Beijing) and Springer-Verlag Berlin Heidelberg, 1998, p. 16.

43. Enderlein R., Phys. Rev. B, 1993, 47, 2162.

44. Huang K., Zhu B.F., Phys. Rev. B, 1988, 38, 2183.

45. Lambin Ph., Senet P., Lucas A.A., Phys. Rev. B, 1991, 44, 6416.

46. Rossi F., Rota L., Bungaro C., Lugli P., Molinari E., Phys. Rev. B, 1993, 47, 1695.

\title{
Стани полярних інтерфейсних оптичних фононів у квантовій точці вюрциту GaN та їхні дисперсійні властивості: вплив квантового розміру
}

\author{
Л. Жанг \\ Політехніка Гуанчжоу Панью, Гуанчжоу 511483, Народна Республіка Китай
}

\begin{abstract}
На основі макроскопічної діелектричної неперервної моделі отримано і досліджено інтерфейсні оптичні пропагаторні (IO-PR) змішані фононні моди циліндричної квантової точки (QD) структури вюрциту квазінульової вимірності (QOD). Даються аналітичні фононні стани IO-PR змішаних мод. Знайдено, що є два типи IO-PR змішаних фононних мод, а саме, $\rho$-IO/z-PR змішані моди і $z$-IO/ $\rho$-PR змішані моди, що існують у квантових точках Q0D вюрциту. Кожна IO-PR мода має симетричну та антисиметричну форми. За допомогою стандартної процедури квантування отримано гамільтоніан Фрьоліха для взаємодії електрон-(IO-PR) змішані фонони. Продемонстровано також ортогональні співвідношення поляризаційних власних векторів для цих IO-PR змішаних мод. Числові обчислення для циліндричної QD вюрциту GaN зосереджуються на впливі квантового розміру на дисперсійні властивості IO-PR змішаних мод. Результати показують, що як радіально напрямлений розмір, так i аксіально напрямлений розмір мають великий вплив на дисперсійні частоти IO-PR змішаних фононних мод. Детально обговорено обмеження властивостей дисперсійних кривих цих фононних мод. Фононні моди, що "редукують" поведінку квантово обмежених структур вюрциту були явно спостережені у цих системах. Крім того, така поведінка, що редукує IO-PR фононні моди у квантових точках вюрциту Q0D до IO мод і PR мод у системах вюрциту Q2D QW and Q1D QWR є глибоко проаналізована з точки зору фізики і математики. Ці результати показують, що дана теорія полярних змішаних фононних мод у циліндричних квантових точках вюрциту узгоджується з теоріями фононних мод для систем вюрциту QWs i QWR. Отриманий аналітичний гамільтоніан електрон-фононної взаємодії є корисним при наступному аналізі фононного впливу на оптоелектронні властивості квантової точки структур вюрциту QOD.
\end{abstract}

Ключові слова: вюрцит нітридні квантові точки, фононні стани, електро-фононні взаємодії, вплив квантового розміру 